\title{
A Lightweight Single-Camera Polarization Compass with Covariance Estimation
}

\author{
Wolfgang Stürzl \\ Institute of Robotics and Mechatronics, German Aerospace Center (DLR) \\ wolfgang.stuerzl@dlr.de
}

\begin{abstract}
A lightweight visual compass system is presented as well as a direct method for estimating sun direction and its covariance. The optical elements of the system are described enabling estimation of sky polarization in a FOV of approx. $56^{\circ}$ with a single standard camera sensor. Using the proposed direct method, the sun direction and its covariance matrix can be estimated based on the polarization measured in the image plane. Experiments prove the applicability of the polarization sensor and the proposed estimation method, even in difficult conditions. It is also shown that in case the sensor is not leveled, combination with an IMU allows to determine all degrees of orientation. Due to the low weight of the sensor and the low complexity of the estimation method the polarization system is well suited for MAVs which have limited payload and computational resources. Furthermore, since not just the sun direction but also its covariance is estimated an integration in a multisensor navigation framework is straight forward.
\end{abstract}

\section{Introduction}

Scattering of sun light in the atmosphere creates a characteristic polarization pattern in the sky that is symmetric with respect to the position of the sun. The degree of polarization increases with angular distance from the sun up to $90^{\circ}$ and decreases for larger angles $[4,13]$. The orientation of the predominantly linearly polarized light is perpendicular to the observer-sun axis. If the expected sun position in a geo-referenced frame for given date and location is known, the estimated position of the sun can be used as an absolute compass. Having some kind of compass is essential for long range path integration or odometry as otherwise small errors in the estimated heading direction soon add up, rendering any directed navigation impossible [3].

Honey-bees and many other insect species, like desert ants, are known to have a specialized region in the upper part of their eyes with photoreceptors sensitive to polarization and it has been shown that they use the polarization pattern of the sky as a compass cue [11]. The indirect ap- proach based on the sky polarization pattern can estimate sun direction even if the sun itself is not visible, e.g. due to occlusion by clouds or trees.

Compass systems based on the polarization have been used on air planes traveling along or close to the north pole. In this region polarization information is particularly useful during winter as the polarization pattern is strongest $90^{\circ}$ away from the sun and continues to be detectable after sun set, i.e. when sun is below the horizon. It has even been discussed whether Vikings could have used sky polarization to guide navigation on open sea [8].

Having a compass system that operates independent of the magnetic field of the earth is also advantageous for mobile robots, since magnetic compasses can be easily be disturbed by nearby magnetic objects or electrical devices.

\section{Previous Work}

In recent years, a number of devices have been built that allow to measure polarization of sky light [12]. Those actually used on robots have usually small FOV and estimate sun azimuth only [5, 2, 22]. Horváth et al. [10] proposed a full-sky polarization sensor consisting of three cameras. Another three-camera system having smaller FOV was described in [22]. A considerably smaller four-camera system has been presented in [20]. The use of multiple cameras make such devices heavy and expensive. Furthermore, cameras have to be synchronized to be applicable to moving platforms. Alternatively, cameras with builtin beam splitters and multiple sensors have been proposed [18], which are usually expensive and bulky. Also, special image sensors have been designed that have pixels with different polarization sensitivity [17, 12]. In contrast, the device presented in this paper is based on a single standard VGA CMOS sensor. While there are several alternative approaches for obtaining polarization information with a single camera sensor, e.g. [9, 15, 23], a particularly small and lightweight solution is described in the following section.

The proposed direct method for determining the direction of the sun improves the approach of [20] by removing the bias and, more importantly, provides an estimate of the covariance matrix. 

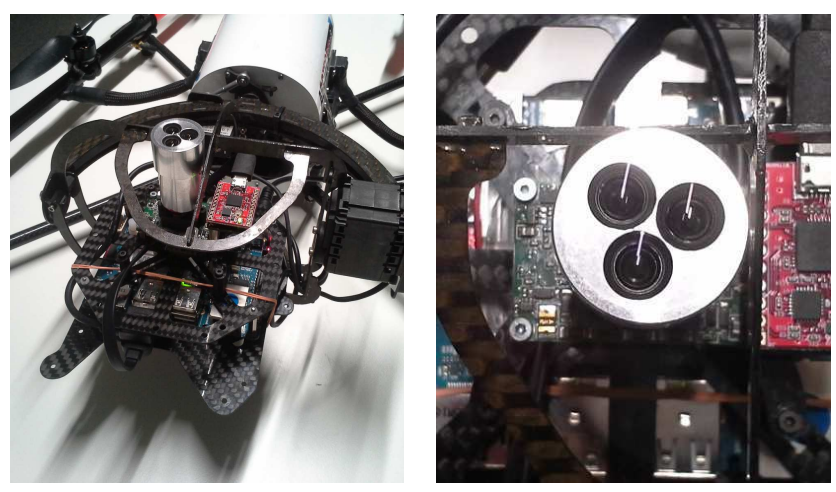

Figure 1. The polarization camera mounted on an AscTec Falcon 8. The camera and the small red circuit board containing an IMU (myAHRS+) are connected to an ODROID-XU3 (Hardkernel).

\section{Polarization Sensor}

\subsection{Design criteria}

As the polarization pattern of the sky changes smoothly (at least for an unobstructed view of the clear sky) there is no need for very high resolution. Nevertheless, reasonably resolution is advantageous in situations where, for instance, trees or buildings leave only small patches of clear sky and averaging over clear sky and non-sky regions would result in poor local estimates of sky polarization. For similar reasons and in order to estimate the full sun vector, i.e., both sun azimuth and elevation, the field of view should not be small. This is particularly important if it cannot be assumed that the sensor is oriented vertically, e.g. stabilized by a gimbal, when used on a highly dynamic platform. For application on a Micro Aerial Vehicle (MAV) with very limited payload low weight and small size are key features.

As described in the following these criteria were met by using a VGA resolution camera sensor with 3 lenses, each lens with a linear polarizer of different orientation attached. The polarization camera is lightweight (about $28 \mathrm{~g}$ for lenses and housing, including camera sensor board) and can be easily carried by MAVs, see Fig. 1 .

\subsection{Optical setup}

The polarization sensor basically consists of a standard board level computer vision camera (PtGrey, now FLIR, Firefly MV 0.3 MP Color USB 2.0, max. resolution $752 \times$ 480 pixels) with a $f=5 \mathrm{~mm}$ S-mount lens and a small custom-built cylindrical optical assembly (see Fig. 2). The latter includes three plano-concave lenses with differently oriented linear polarizers (orientations differ by $120^{\circ}$ ) and a biconvex lens with $f=30 \mathrm{~mm}$ focal length, equal to its distance to the camera lens. This arrangement results in three almost identical images on the camera sensor. Note, that the focal plane is not exactly the same for all rays, which

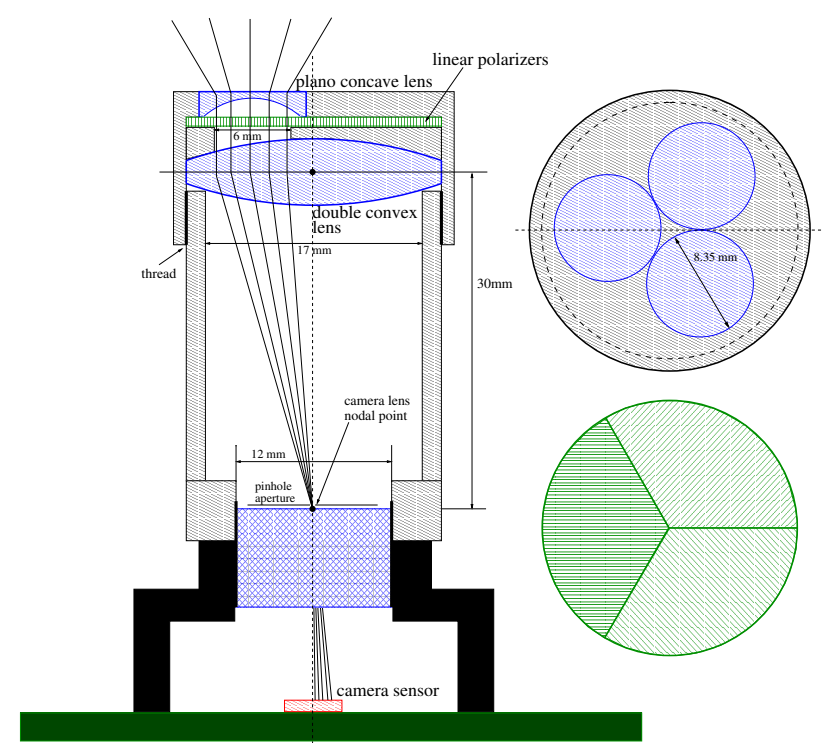

Figure 2. Schematic drawing of the imaging system. Differently oriented linear polarizers (green) are located behind three planoconcave lenses. The camera lens is in the focal plane of the double convex lens (focal length $30 \mathrm{~mm}$, diameter $20 \mathrm{~mm}$ ) which causes principal rays through the nodal point of the camera lens to run parallel between the biconvex lens and the plano-concave lenses. The camera lens is an off-the-shelf $5 \mathrm{~mm} \mathrm{~S}$-mount lens.

could produce blurred images. This effect is ameliorated by a small aperture of the camera lens (pinhole aperture).

A debayered but otherwise unprocessed image recorded with the polarization camera is shown in Fig. 3 a.

\subsection{Calibration and Remapping}

The sub-cameras, i.e. the optical pathways creating the three sub-images, were calibrated with the DLR-CalDe/ CalLab tool [19] based on a pinhole camera model with two radial distortion parameters. Using the calibration results the three sub-images can be remapped in a way that
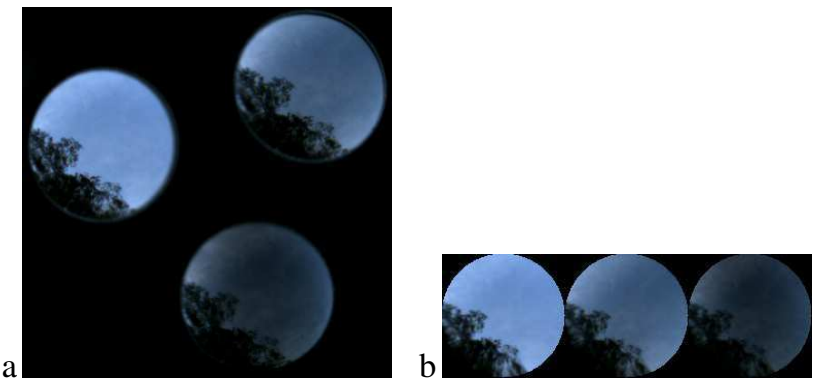

Figure 3. Remapping of an original RGB image (size $408 \times 408$ pixels) shown in (a) to three $120 \times 120$ images with $0.5^{\circ}$ per pixel resolution (b), i.e. the elevation angle decreases linearly from $90^{\circ}$ (center) to $60^{\circ}$ (periphery) along radial lines (assuming the camera axis is aligned with gravity and lenses are viewing the sky). 
projects distant objects (objects with distance to the camera $\gg$ distance between the centers of the plano-concave lenses, approx. $8 \mathrm{~mm}$ ) to the same pixel coordinates in each of the three resulting images, i.e. corresponding pixels in each of the three images cover the same solid angle on the viewing sphere, see Fig. 3 b. A projection with constant radial resolution of res $=0.5^{\circ} / \mathrm{px}$ was selected. The mapping between a point in one of the three polarization images with coordinates $\left(u_{i}, v_{i}\right)$ and the viewing direction $\mathbf{e}_{i}$ is described by

$$
\begin{aligned}
\mathbf{e}_{i} & =\left(\cos \operatorname{azi}_{i} \cos \mathrm{ele}_{i}, \sin _{\mathrm{azi}} \cos \mathrm{ele}_{i}, \sin _{\mathrm{ele}}\right)^{\top}, \\
\mathrm{azi}_{i} & =\arctan 2\left(v_{i}-v_{c}, u_{i}-u_{c}\right) \\
\text { ele }_{i} & =\frac{\pi}{2}-\operatorname{res} \sqrt{\left(v_{i}-v_{c}\right)^{2}+\left(u_{i}-u_{c}\right)^{2}} \\
\left(\begin{array}{c}
u_{i} \\
v_{i}
\end{array}\right) & =\left(\begin{array}{l}
u_{c} \\
v_{c}
\end{array}\right)+\left(\begin{array}{c}
\cos \mathrm{azi}_{i} \\
\sin \mathrm{azi}_{i}
\end{array}\right)\left(\frac{\pi}{2}-\mathrm{ele}_{i}\right) / \text { res }
\end{aligned}
$$

where $u_{c}, v_{c}$ are the coordinates of the image center, azi $\mathrm{i}_{\mathrm{i}}$ and ele $e_{i}$ are azimuth and elevation angle of $\mathbf{e}_{i}$.

\subsection{Mapping of polarization pattern to image plane}

Assuming a linear transfer function between the number of absorbed photons (collected electrons) and the pixel value $I$, which is correct for most camera sensors if gamma correction is disabled, then the pixel value for partially linearly polarized light passing a linear polarizer oriented at angle $\phi_{k}$ is given by (see, e.g. [1])

$$
I_{k}(\phi)=I_{0}\left(1+\delta \cos \left(2\left(\phi-\phi_{k}\right)\right)\right) .
$$

$I_{0}$ is the average pixel value, $\delta$ the degree of polarization and $\phi$ the angle of the polarized light. The parameters can be estimated if there are measurements, i.e. pixel values $I_{k}$, for at least three different polarizer angles by minimizing

$$
E\left(I_{0}, \delta, \phi\right)=\sum_{k}\left(I_{0}+I_{0} \delta \cos \left(2\left(\phi-\phi_{k}\right)\right)-I_{k}\right)^{2} .
$$

For $K$ polarizers with angles equally distributed in $[0,2 \pi)$, the solution is

$$
\begin{aligned}
I_{0} & =K^{-1} \sum_{k} I_{k}, \\
\delta & =I_{0}^{-1} \sqrt{S^{2}+C^{2}}, \\
\phi & =\frac{1}{2} \arctan 2(S, C),
\end{aligned}
$$

where $S=\frac{2}{K} \sum_{k} I_{k} \sin \left(2 \phi_{k}\right), C=\frac{2}{K} \sum_{k} I_{k} \cos \left(2 \phi_{k}\right)$.

The polarizer angles $\phi_{k}$ were measured with respect to the camera frame by turning a light source with partially polarized light of known polarization angle. The estimated values are $\phi_{1}=106.7^{\circ}, \phi_{2}=226.7^{\circ}$ and $\phi_{3}=346.7^{\circ}$.

Note that due to the small aperture of the camera lens, vignetting is not of major concern and currently not corrected. Even in case of stronger vignetting, intensities of

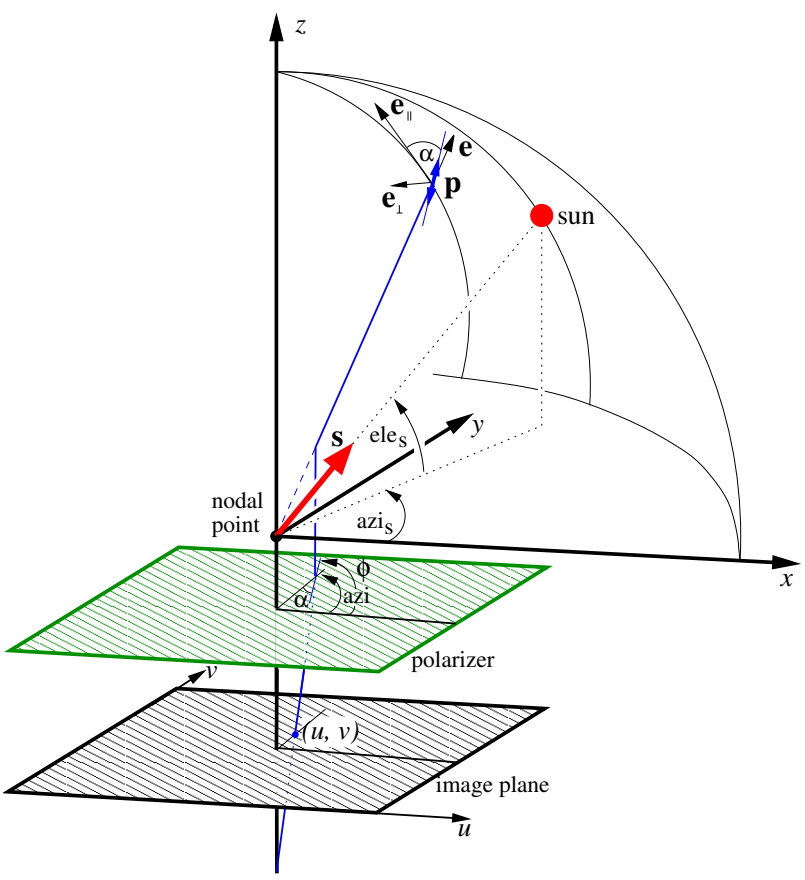

Figure 4. Simplified schematic drawing of the mapping of a point on the "sky sphere" onto the image plane. The $x$ and $y$ axes are parallel to the $u$ and $v$ coordinate axes defining the camera frame. As light is a transverse, electromagnetic wave, the polarization vector $\mathbf{p}$ at a position on the sky sphere is orthogonal to the corresponding direction vector $\mathbf{e}$ and a local coordinate system can be defined using $\mathbf{e}, \mathbf{e}_{\perp}, \mathbf{e}_{\|} ; \mathbf{e}_{\|}$is a unit vector tangent to the great circle through the position and the zenith, pointing upwards, i.e. $\mathbf{e}_{\|}^{\top} \mathbf{e}_{z} \geq 0$, and $\mathbf{e}_{\perp}=\mathbf{e}_{\|} \times \mathbf{e}$. The local sky polarization angle is given by $\alpha=\arccos \left(\mathbf{e}_{\|}^{\top} \mathbf{p}\right)$, i.e. the angle between the polarization vector and the great circle.

corresponding pixels would be reduced by similar factors and degree and angle of polarization would not be affected.

As depicted in Fig. 4 the polarization vector at viewing direction $\mathbf{e}_{i}$ can be expressed as

$$
\mathbf{p}_{i}=\cos \alpha_{i} \mathbf{e}_{\|, i}-\sin \alpha_{i} \mathbf{e}_{\perp, i}
$$

The vectors $\mathbf{e}_{\perp, i}$ and $\mathbf{e}_{\|, i}$ are defined as

$$
\begin{aligned}
\mathbf{e}_{\|, i} & =\frac{\partial}{\partial \mathrm{ele}_{i}} \mathbf{e}_{i}=\left(\begin{array}{r}
-\cos \left(\mathrm{azi}_{i}\right) \sin \left(\mathrm{ele}_{i}\right) \\
-\sin \left(\mathrm{azi}_{i}\right) \sin \left(\mathrm{ele}_{i}\right) \\
\cos \left(\mathrm{ele}_{i}\right)
\end{array}\right), \\
\mathbf{e}_{\perp, i} & =\frac{1}{\cos \left(\mathrm{ele}_{i}\right)} \frac{\partial}{\partial \operatorname{azi}_{i}} \mathbf{e}_{i}=\left(\begin{array}{r}
-\sin \left(\mathrm{azi}_{i}\right) \\
\cos \left(\mathrm{azi}_{i}\right) \\
0
\end{array}\right) .
\end{aligned}
$$

Note that, since polarization has no direction but just orientation, either $\mathbf{p}_{i}$ or $-\mathbf{p}_{i}$ can be used.

From the polarization angle $\phi_{i}$ in the image plane as determined from the three polarization images using Eq. (9), the sky polarization angle $\alpha_{i}$ can be computed (see Fig. 4),

$$
\alpha_{i}=\phi_{i}-\operatorname{azi}_{i} .
$$


Combining Eqs. (9), (10) and (13), the unit polarization vector $\mathbf{p}_{i}=\mathbf{p}\left(u_{i}, v_{i}\right)$ for each viewing direction $\mathbf{e}_{i}=\mathbf{e}\left(u_{i}, v_{i}\right)$ can be calculated from the corresponding pixel values in the three polarization images, $I_{1 i}=I_{1}\left(u_{i}, v_{i}\right), I_{2 i}=I_{2}\left(u_{i}, v_{i}\right)$ and $I_{3 i}=I_{3}\left(u_{i}, v_{i}\right)$.

\section{Estimation of sun vector and its covariance}

In this section a direct method for estimating the sun vector and its covariance matrix is described. The approach of [20] is enhanced by (1) removing the bias and by (2) estimating the covariance matrix, which is essential for assessing the reliability of measurements and for fusion with data from other sensors, e.g. from an IMU.

According to the Rayleigh model, the sun is in the center of the polarization pattern and all polarization vectors $\left\{\mathbf{p}_{i}\right\}_{i=1,2, \ldots, N}$ are perpendicular to the "sun vector" $\mathbf{s}$, a unit vector pointing from the observer to the sun, i.e. $\mathbf{p}_{i}^{\top} \mathbf{s}=$ 0 . In real situations this holds only approximately and one can minimize, under the constraint $\|\mathbf{s}\|=1$, the weighted sum

$$
E(\mathbf{s})=\frac{1}{\sum_{i} w_{i}} \sum_{i} w_{i}\left(\mathbf{p}_{i}^{\top} \mathbf{s}\right)^{2}=\mathbf{s}^{\top} \hat{\mathbf{P}} \mathbf{s}
$$

instead, where $\hat{\mathbf{P}}=\frac{1}{\sum_{i} w_{i}} \sum_{i} w_{i} \mathbf{p}_{i} \mathbf{p}_{i}^{\top}$ is the "polarization matrix". Minimizing Eq. (14) under the constraint $\|\mathbf{s}\|^{2}=1$, which can be expressed using Lagrange multiplier $\lambda$, leads to an eigenvalue problem,

$$
\begin{aligned}
\bar{E}(\mathbf{s}, \lambda) & =\mathbf{s}^{\top} \hat{\mathbf{P}} \mathbf{s}-\lambda\left(\mathbf{s}^{\top} \mathbf{s}-1\right), \\
\mathbf{0} & =\nabla_{\mathbf{s}} \bar{E}(\mathbf{s}, \lambda)=\hat{\mathbf{P}} \mathbf{s}-\lambda \mathbf{s}, \\
\rightsquigarrow \quad \hat{\mathbf{P}} \mathbf{s} & =\lambda \mathbf{s},
\end{aligned}
$$

and the sun vector $\mathbf{s}$ is the eigenvector of matrix $\hat{\mathbf{P}}$ corresponding to the smallest eigenvalue. So far, the approach of [20] has been summarized and the notation introduced.

Before describing the main contributions of the paper for enhancing sun vector estimation it should be noted that $E$ (s) defined in Eq. (14) can also be interpreted as the (weighted) average squared deviation angle $\left\langle\Delta^{2}\right\rangle$ from the ideal $90^{\circ}$ between the sun vector and the polarization vectors,

$$
\begin{aligned}
E(\mathbf{s}) & =\frac{1}{\sum_{i} w_{i}} \sum_{i} w_{i} \cos ^{2} \angle\left(\mathbf{s}, \mathbf{p}_{i}\right) \\
& =\frac{1}{\sum_{i} w_{i}} \sum_{i} w_{i} \cos ^{2}\left(\pi / 2-\Delta_{i}\right) \\
& =\frac{1}{\sum_{i} w_{i}} \sum_{i} w_{i} \sin ^{2} \Delta_{i} \\
\stackrel{\Delta_{i} \mid \ll 1}{\approx} & \frac{1}{\sum_{i} w_{i}} \sum_{i} w_{i} \Delta_{i}^{2}=\left\langle\Delta^{2}\right\rangle .
\end{aligned}
$$

\subsection{Bias removal}

Solving Eq. (17) can result in biased estimates of the sun vector as shown in the following. Considering the simplest case of independent and identically distributed zero-mean noise added to each component of the "true" polarization vectors $\mathbf{p}_{0 i}$, i.e. assuming the measured polarization vectors to be $\mathbf{p}_{i}=\mathbf{p}_{0 i}+\boldsymbol{\Delta} \mathbf{p}_{i}$, the expected polarization matrix is

$$
\begin{aligned}
\langle\hat{\mathbf{P}}\rangle & =\sum_{i} \tilde{w}_{i}\left\langle\left(\mathbf{p}_{0 i}+\boldsymbol{\Delta} \mathbf{p}_{i}\right)\left(\mathbf{p}_{0 i}+\boldsymbol{\Delta} \mathbf{p}_{i}\right)^{\top}\right\rangle \\
& =\sum_{i} \tilde{w}_{i} \mathbf{p}_{0 i} \mathbf{p}_{0 i}^{\top}+\sum_{i} \tilde{w}_{i}\left\langle\boldsymbol{\Delta} \mathbf{p}_{i} \boldsymbol{\Delta} \mathbf{p}_{i}^{\top}\right\rangle \\
& \stackrel{* *)}{=} \hat{\mathbf{P}}_{0}+\sigma^{2} \hat{\mathbf{n}}
\end{aligned}
$$

where $\tilde{w}_{i}=\frac{w_{i}}{\sum_{i} w_{i}} \cdot \hat{\mathbf{P}}_{0}=\sum_{i} \tilde{w}_{i} \mathbf{p}_{0 i} \mathbf{p}_{0 i}^{\top}$ is the error-free polarization matrix and $\hat{\mathbf{n}}=\sum_{i} \tilde{w}_{i}\left(\hat{\mathbf{1}}-\mathbf{e}_{i} \mathbf{e}_{i}^{\top}\right)$ the noise matrix that leads to a biased estimate, unless it is proportional to the identity matrix. The transformation (*) is based on the fact that due to the transverse polarization of light the polarization vectors $\mathbf{p}_{i}$ and therefore also the error vectors $\Delta \mathbf{p}_{i}$ are orthogonal to the viewing direction $\mathbf{e}_{i}$.

As an informative example, matrix $\hat{\mathbf{n}}$ can be computed for the special case $w_{i}=1 \forall i$ in dependence on the FOV of the camera, defined by parameter $2 \theta$. With the leveled camera pointing upwards this means that the minimum elevation angle is ele $\mathrm{e}_{\min }=90^{\circ}-\theta$. Approximating the sum by an integral in spherical coordinates and assuming equally distributed viewing directions on the sphere, described by density function $D($ azi, ele $)=\frac{1}{2 \pi}$ cos ele, leads to

$$
\begin{aligned}
\hat{\mathbf{n}} & =\frac{1}{N} \sum_{i}\left(\hat{\mathbf{1}}-\mathbf{e}_{i} \mathbf{e}_{i}^{\top}\right)=\left(\begin{array}{ccc}
A & 0 & 0 \\
0 & A & 0 \\
0 & 0 & B
\end{array}\right), \\
A & =\frac{1}{6}\left(4+\sin \text { ele }_{\max }+\sin ^{2} \text { ele }_{\min }\right) \\
& =\frac{1}{6}\left(4+\cos \theta+\cos ^{2} \theta\right) \\
B & =\frac{1}{3}\left(2-\sin \text { ele }_{\min }-\sin ^{2} \text { ele }_{\min }\right) \\
& =\frac{1}{3}\left(2-\cos \theta-\cos ^{2} \theta\right)
\end{aligned}
$$

As shown in Fig. 5, the difference of $A(\theta)$ and $B(\theta)$ is more pronounced, and a bias reduction therefore more important, for small FOV.

\subsubsection{Pre-whitening of the polarization matrix}

If a symmetric (positive definite) matrix $\hat{\mathbf{P}}$ is the sum of two symmetric matrices, $\hat{\mathbf{P}}=\hat{\mathbf{P}}_{o}+\hat{\Delta \mathbf{n}}$, where $\hat{\Delta \mathbf{n}}$ contains non-zero-mean noise, so that $\langle\hat{\mathbf{P}}\rangle=\hat{\mathbf{P}}_{o}+\sigma^{2} \hat{\mathbf{n}}$, then, as described by [16], matrix pre-whitening [14] can be used in 


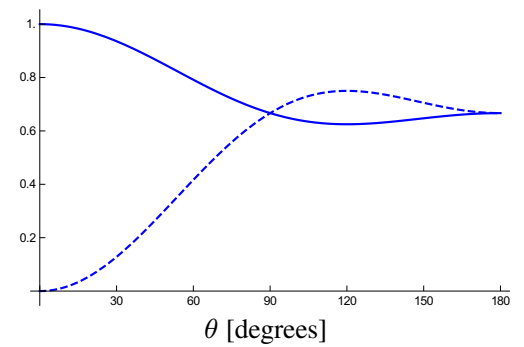

Figure 5. Diagonal elements $A$ (solid curve) and $B$ (dashed), see Eqs. (27) and (29), of the bias matrix in dependence on angle $\theta$, which is half of the FOV. For $\theta=90^{\circ}\left(2 \theta=180^{\circ} \mathrm{FOV}\right)$ the two curves intersect and their difference stays small for even larger FOV.

order to eliminate the bias when estimating eigenvectors by introducing $\hat{\tilde{\mathbf{P}}}=\hat{\mathbf{n}}^{-1 / 2} \hat{\mathbf{P}} \hat{\mathbf{n}}^{-1 / 2}$.

From the eigenvalues $\tilde{\lambda}$ and eigenvectors $\tilde{\mathbf{v}}$ of $\langle\hat{\tilde{\mathbf{P}}}\rangle=$ $\hat{\mathbf{n}}^{-1 / 2} \hat{\mathbf{P}}_{o} \hat{\mathbf{n}}^{-1 / 2}+\sigma^{2} \hat{\mathbf{I}}$, defined by solving

$$
\langle\hat{\tilde{\mathbf{P}}}\rangle \tilde{\mathbf{v}}=\tilde{\lambda} \tilde{\mathbf{v}}
$$

one can calculate the eigenvectors of matrix $\hat{\mathbf{n}}^{-1} \hat{\mathbf{P}}_{o}$ to be $\hat{\mathbf{n}}^{-1 / 2} \tilde{\mathbf{v}}$ :

$$
\begin{aligned}
\langle\hat{\tilde{\mathbf{P}}}\rangle \tilde{\mathbf{v}}=\hat{\mathbf{n}}^{-1 / 2} \hat{\mathbf{P}}_{o} \hat{\mathbf{n}}^{-1 / 2} \tilde{\mathbf{v}}+\sigma^{2} \tilde{\mathbf{v}} & =\tilde{\lambda} \tilde{\mathbf{v}} \\
& =\left(\lambda_{0}+\sigma^{2}\right) \tilde{\mathbf{v}} \\
\Longleftrightarrow \hat{\mathbf{n}}^{-1 / 2}\left(\hat{\mathbf{n}}^{-1 / 2} \hat{\mathbf{P}}_{o} \hat{\mathbf{n}}^{-1 / 2} \tilde{\mathbf{v}}\right) & =\hat{\mathbf{n}}^{-1 / 2}\left(\lambda_{0} \tilde{\mathbf{v}}\right)
\end{aligned}
$$

Note that $\hat{\mathbf{n}}^{-1 / 2} \tilde{\mathbf{v}}$ is eigenvector of $\hat{\mathbf{n}}^{-1} \hat{\mathbf{P}}_{o}$ and, in general, not of $\hat{\mathbf{P}}_{o}$. However, as stated in [14], since $\hat{\mathbf{P}}_{0}$ is noisefree, its minimum eigenvalue is 0 and thus the corresponding eigenvector is also eigenvector of matrix $\hat{\mathbf{n}}^{-1} \hat{\mathbf{P}}_{o}$ for eigenvalue 0 . In other words, $\hat{\mathbf{n}}^{-1} \hat{\mathbf{P}}_{o}$ and $\hat{\mathbf{P}}_{o}$ have the same eigenvector for eigenvalue 0 . Also note, that $\hat{\mathbf{n}}^{-1} \hat{\mathbf{P}}_{0}$ is not a symmetric matrix and, thus, its eigenvectors do not form an orthogonal basis, i.e. while the eigenvectors of $\hat{\tilde{\mathbf{P}}}, \tilde{\mathbf{v}}, \tilde{\mathbf{v}}_{1}$ and $\tilde{\mathbf{v}}_{2}$ are orthogonal, $\hat{\mathbf{n}}^{-1 / 2} \tilde{\mathbf{v}}, \hat{\mathbf{n}}^{-1 / 2} \tilde{\mathbf{v}}_{1}$ and $\hat{\mathbf{n}}^{-1 / 2} \tilde{\mathbf{v}}_{2}$ are not.

\subsection{Covariance of estimated sun vector}

In the following the covariance matrix $\left\langle\Delta \mathbf{s} \Delta \mathbf{s}^{\top}\right\rangle$ will be derived for the unbiased estimate of the sun vector $\tilde{\mathbf{s}}=\frac{1}{\left\|\hat{\mathbf{n}}^{-1 / 2} \tilde{\mathbf{v}}\right\|} \hat{\mathbf{n}}^{-1 / 2} \tilde{\mathbf{v}}$, where $\tilde{\mathbf{v}}$ is the eigenvector corresponding to the smallest eigenvalue $\tilde{\lambda}$ of matrix $\hat{\tilde{\mathbf{P}}}=$ $\hat{\mathbf{n}}^{-1 / 2} \hat{\mathbf{P}} \hat{\mathbf{n}}^{-1 / 2}=\hat{\mathbf{n}}^{-1 / 2}\left(\hat{\mathbf{P}}_{0}+\hat{\boldsymbol{\Delta}} \mathbf{n}\right) \hat{\mathbf{n}}^{-1 / 2}=\hat{\tilde{\mathbf{P}}}_{0}+\hat{\tilde{\Delta}} \mathbf{n}$. Due to $\hat{\tilde{\Delta}} \mathbf{n}, \tilde{\mathbf{v}}$ deviates from eigenvector $\tilde{\mathbf{v}}_{0}$ of $\hat{\tilde{\mathbf{P}}}_{0}$ corresponding to eigenvalue 0 , i.e. $\tilde{\mathbf{v}}=\tilde{\mathbf{v}}_{0}+\Delta \tilde{\mathbf{v}}$. Adapting the approach of [21] the error vector in first order approximation is given by

$$
\begin{aligned}
\boldsymbol{\Delta} \tilde{\mathbf{v}} & \approx \hat{\tilde{\mathbf{M}} \boldsymbol{\tilde { \mathbf { \Delta } }} \mathbf{n} \tilde{\mathbf{v}}} \\
\hat{\tilde{\mathbf{M}}} & =-\frac{1}{\tilde{\lambda}_{1}-\tilde{\lambda}} \tilde{\mathbf{v}}_{1} \tilde{\mathbf{v}}_{1}^{\top}-\frac{1}{\tilde{\lambda}_{2}-\tilde{\lambda}} \tilde{\mathbf{v}}_{2} \tilde{\mathbf{v}}_{2}^{\top} .
\end{aligned}
$$

$\tilde{\mathbf{v}}_{1}$ and $\tilde{\mathbf{v}}_{2}$ are the eigenvectors of $\hat{\tilde{\mathbf{P}}}$ orthogonal to $\tilde{\mathbf{v}}, \tilde{\lambda}_{1}$ and $\tilde{\lambda}_{2}$ the corresponding eigenvalues $\left(\lambda_{1}, \lambda_{2}>\lambda\right)$. Note that the error vector $\Delta \tilde{\mathbf{v}}$ is orthogonal to $\tilde{\mathbf{v}}$.

Starting from $\frac{\hat{\mathbf{n}}^{-1 / 2}(\tilde{\mathbf{v}}+\Delta \tilde{\mathbf{v}})}{\left\|\hat{\mathbf{n}}^{-1 / 2}(\tilde{\mathbf{v}}+\Delta \tilde{\mathbf{v}})\right\|}=\tilde{\mathbf{s}}+\Delta \mathbf{s}$, the error of the sun vector is

$$
\Delta \mathbf{s} \approx\left(\hat{\mathbf{1}}-\tilde{\mathbf{s}} \tilde{\mathbf{s}}^{\top}\right) \frac{\hat{\mathbf{n}}^{-1 / 2} \Delta \tilde{\mathbf{v}}}{\left\|\hat{\mathbf{n}}^{-1 / 2} \tilde{\mathbf{v}}\right\|} .
$$

Combining Eqs. (34) and (36), the covariance matrix can be approximated (after some algebraic manipulations) by

$$
\left\langle\boldsymbol{\Delta} \mathbf{s} \Delta \mathbf{s}^{\top}\right\rangle \approx \hat{\mathbf{Q}}_{\tilde{\mathbf{s}}}\left\langle\hat{\mathbf{\Delta}} \mathbf{\mathbf { s }} \tilde{\mathbf{s}} \tilde{\mathbf{s}}^{\top} \hat{\Delta \mathbf{n}}\right\rangle \hat{\mathbf{Q}}_{\tilde{\mathbf{s}}}^{\top}
$$

where $\hat{\mathbf{Q}}_{\tilde{\mathbf{s}}}=\left(\hat{\mathbf{1}}-\tilde{\mathbf{s}} \tilde{\mathbf{S}}^{\top}\right) \hat{\mathbf{n}}^{-1 / 2} \hat{\tilde{\mathbf{M}}} \hat{\mathbf{n}}^{-1 / 2}$.

Assuming independent and identically distributed errors in the components of the polarization vector, it can be shown that

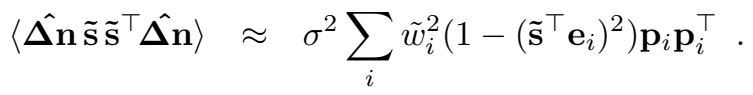

\subsection{Weighting scheme}

In order to obtain robust estimates of sun vector and covariance matrix, it is important to provide suitable weights $w_{i}$. Currently, the following heuristic weighting scheme is used:

$$
\begin{aligned}
w_{i} & =w_{i}^{\mathrm{pol}} \cdot w_{i}^{\mathrm{sat}} \cdot w_{i}^{\nu} \\
w_{i}^{\mathrm{pol}} & =\min \left(I_{0 i} \delta_{i},\left(I_{0} \delta\right)_{\max }\right)^{2} \Theta(1-\delta), \\
w_{i}^{\mathrm{sat}} & =\prod_{k=1}^{3} \Theta\left(I_{\mathrm{sat}}-I_{k i}\right)
\end{aligned}
$$

where $\Theta($.$) is the Heaviside step function. The rationale$ behind $w_{i} \propto\left(I_{0 i} \delta_{i}\right)^{2}$ for $I_{0 i} \delta_{i}<\left(I_{0} \delta\right)_{\max }$, Eq. (40), is that polarization vectors can be estimated more reliably for both larger degree of polarization and average intensity since $I_{0 i} \delta_{i}$ is the intensity amplitude due to polarized light, see Eq. (5). (Note that $I_{0 i} \delta_{i}=\sqrt{S_{i}^{2}+C_{i}^{2}}$, see Eq. (8)). The threshold value $\left(I_{0} \delta\right)_{\max }$ avoids single outliers having extremely high weights. Outliers can be, for instance, due to very close objects (see below) or simply dirt on one of the lenses. The factor $\Theta(1-\delta)$ excludes "unphysical" degrees of polarization estimated using Eq. (8). $\delta>1$ can happen for very close objects, where, because of the distance between the three lenses, intensity differences are not due to polarization but due to viewing slightly different points in space. Note that since there are just three measurements $\left(I_{1 i}, I_{2 i}, I_{3 i}\right)$ for each direction $i$, all parameter values estimated using Eqs. (7)-(9) will result in $E\left(I_{0}, \delta, \phi\right)$, Eq. (6), being zero. For more than $K=3$ measurements, e.g. when using 4 lenses with different polarizer orientation, a large error $E\left(I_{0}, \delta, \phi\right)$ could reveal such issues more reliably. 
Eq. (41) excludes over-exposed pixels from the estimation. The third factor in Eq. (39), $w_{i}^{\nu}$, is based on the idea that the red channel value of a RGB pixel should not be significantly higher than the blue channel value for patches of clear sky. In the current implementation $w_{i}^{\nu}=$ $\frac{1}{2}+\frac{1}{2} \tanh \left(\alpha \frac{B_{i}-\left(R_{i}+\beta\right)}{B_{i}+\left(R_{i}+\beta\right)}\right)$ is used, with $\alpha=10$ and $\beta=1$. $B_{i}$ and $R_{i}$ are the blue and red channel values of pixel $i$. $\left(I_{0} \delta\right)_{\max }$ was set to 100 and $I_{\text {sat }}=255$ as the camera images have 8 bit per pixel.

\subsection{Estimation of noise level}

As an estimate for the "noise level" $\sigma$ in Eq. (38) one could take the square root of the smallest eigenvalue of $\hat{\tilde{\mathbf{P}}}$ since

$$
\sqrt{\lambda}=\sqrt{\tilde{\mathbf{v}}^{\top} \hat{\tilde{\mathbf{P}}} \tilde{\mathbf{v}}} \approx \sqrt{\sigma^{2} \tilde{\mathbf{v}}^{\top} \hat{\mathbf{I}} \tilde{\mathbf{v}}}=\sigma
$$

In order to account for deviations from the independently and identically distributed error model, Eq. (42) has to be extended and the heuristic approach

$$
\sigma=C_{0} \sqrt{\left(1+\frac{C_{1}}{\sum_{i} w_{i}}\right) \lambda}
$$

is used. The parameters are set to $C_{0}=10$ and $C_{1}=$ $10^{6}$, which is about $1 \%$ of the maximum sum of weights $\left(\sum_{i} \max \left(w_{i}\right)=\sum_{i}\left(I_{0} \delta\right)_{\max }^{2} \approx 10^{8}\right)$. This is motivated by the fact that larger deviations from the true sun vector are more likely if the sum of weights is low.

\section{Experiments and results}

For the experiments described in this section, $I_{1 i}, I_{2 i}, I_{3 i}$ were set to the blue channel of the three remapped images.

Exposure control. In order to avoid larger regions of the image being over- or underexposed a simple exposure control was realized. Note that since the 3 sub-images cover only part of the sensor, the built-in auto-exposure of the camera can not be used as it would result in overexposed polarization images. The exposure control tries to ensure that only $1 \%$ of pixels in the 3 sub-images have values equal to $I_{\text {sat }}$. In case of more than $1 \%$ overexposed pixels the exposure time is reduced by factor 0.5 , otherwise the maximum pixel value $I_{\max }$ is examined. If it is $10 \%$ below a certain threshold $I_{\mathrm{th}}$ (250 in the current implementation) the exposure time will be set to $T_{\exp }^{\prime}=I_{\mathrm{th}} / I_{\max } T_{\exp }$, where $T_{\exp }$ is the current exposure time of the camera.

\subsection{Test on a turn table under clear sky}

In the first test, the polarization camera was mounted on a turn-table and rotated in steps of about 30 degrees. Data was recorded under clear sky in the evening with sun at $19^{\circ}$ elevation angle. Sun vectors were estimated with and without bias removal for the full $56^{\circ}$ FOV of the polarization system and for a reduced FOV of just $5^{\circ}$, where only the central pixels (corresponding to elevation angles $\geq 87.5^{\circ}$ ) were used, see Fig. 6. Standard deviations for azimuth and elevation angle were calculated from the estimated covariance matrices by determining the width of the "error ellipse" along horizontal and vertical axes, which in most cases were close to the minor and major axes of the ellipse. The error ellipse is the projection of the covariance matrix onto the plane orthogonal to the estimated sun vector $\tilde{\mathbf{s}}$. Note that $\tilde{\mathbf{s}}$ is eigenvector of the covariance matrix for eigenvalue 0, see Eq. (37).

The results in Fig. 6 a demonstrate that the described bias reduction can significantly improve the estimation of the sun vector, particularly the sun elevation angle, for small FOV. For $5^{\circ}$ FOV, the median deviation of the estimated from the true elevation angle of the sun could by reduced by more than $50 \%$ (from approx $10^{\circ}$ without to approx $4.5^{\circ}$ with bias reduction). However for the full $56^{\circ} \mathrm{FOV}$ of the polarization sensor the improvement due to bias reduction is negligible under these ideal conditions. While the estimated azimuth angle follows the $30^{\circ}$ steps very reliably even for small FOV, the estimation of the elevation angle benefits from larger FOV.

\subsection{Test in complex scene, with tilting and IMU}

While only the azimuth angle of the sun is needed on a leveled platform to provide compass information, the situation is more complex on a tilted platform or even on a highly dynamic system like a MAV. Since the polarization pattern is symmetric with respect to the sun, the sun vector on its own is not sufficient to provide meaningful orientation information. However if the tilt angle is known, e.g. by visual detection of the skyline or from measurements of an IMU, all rotational DOFs can be determined, in case the full sun vector (azimuth and elevation angle) has been determined.

In the second test, where the polarization sensor was turned around horizontal axes, measurements of an attached IMU (see Fig. 1) were used to compensate for tilt. If the rotation between the camera and the IMU described by matrix $\hat{\mathbf{R}}_{\mathrm{IMU}}^{\mathrm{cam}}$ is known, e.g. estimated using an IMU to camera calibration procedure $[6,7]$, then the transformed sun vector in the leveled reference frame is given by

$$
\begin{aligned}
\mathbf{s}_{\mathrm{lev}} & =\hat{\mathbf{R}}_{\mathrm{lev}}^{\mathrm{cam}} \mathbf{s}_{\mathrm{cam}}, \\
\hat{\mathbf{R}}_{\mathrm{lev}}^{\mathrm{cam}} & =\left(\hat{\mathbf{R}}_{\mathrm{IMU}}^{\mathrm{cam}}\right)^{\top} \hat{\mathbf{R}}_{\mathrm{pitch}} \hat{\mathbf{R}}_{\mathrm{roll}} \hat{\mathbf{R}}_{\mathrm{IMU}}^{\mathrm{cam}} .
\end{aligned}
$$

$\hat{\mathbf{R}}_{\text {pitch }}$ and $\hat{\mathbf{R}}_{\text {roll }}$ are the roll and pitch rotation matrices in the IMU frame. In case uncertainties due to the IMU measurements can be neglected, the covariance matrix can be transformed accordingly,

$$
\left\langle\boldsymbol{\Delta} \mathbf{s} \boldsymbol{\Delta} \mathbf{s}^{\top}\right\rangle_{\text {lev }}=\hat{\mathbf{R}}_{\mathrm{lev}}^{\mathrm{cam}}\left\langle\boldsymbol{\Delta} \mathbf{s} \boldsymbol{\Delta} \mathbf{s}^{\top}\right\rangle_{\mathrm{cam}}\left(\hat{\mathbf{R}}_{\mathrm{lev}}^{\mathrm{cam}}\right)^{\top}
$$


FOV $5^{\circ}$

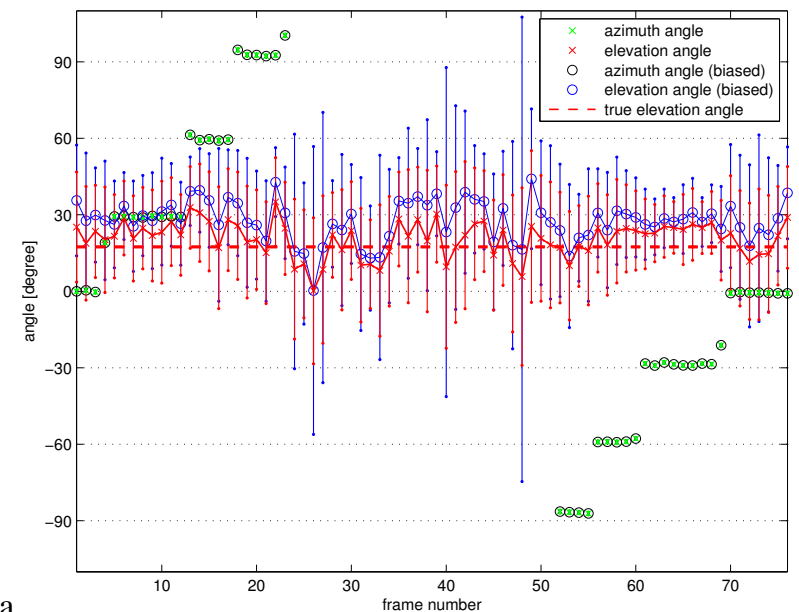

FOV $56^{\circ}$

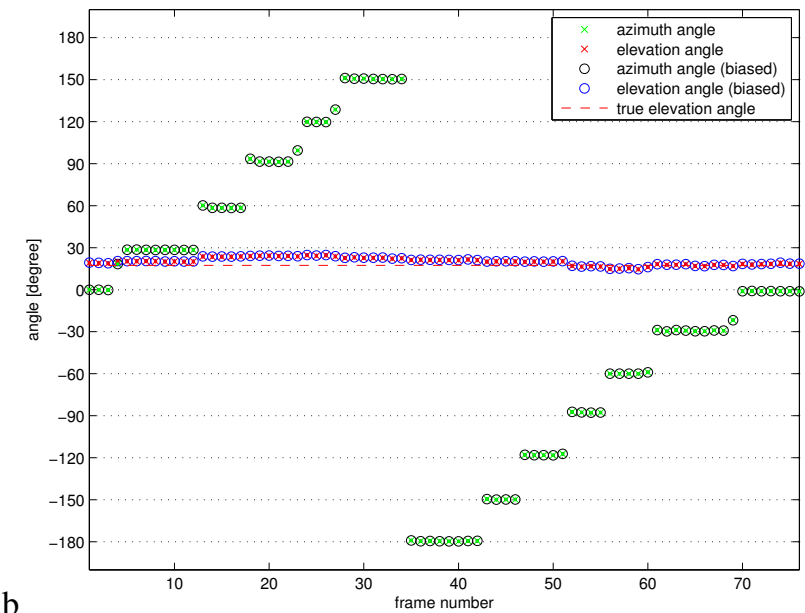

Figure 6. Determining sun direction with and without bias removal on data recorded during a $360^{\circ}$ rotation in steps of $30^{\circ}$ under a clear sky. Shown are estimates of sun azimuth and elevation angles and standard deviations (depicted as vertical lines of same color). a) Bias removal significantly enhances accuracy of elevation angle estimation for a small $5^{\circ} \mathrm{FOV}$; compare points in red and blue (biased estimator) for elevation angle and in green and black (biased estimator) for azimuth. Note that the range of the $y$-axis has been limited to $\left[-100^{\circ}, 100^{\circ}\right]$ to enhance visibility. b) The gain in accuracy using bias removal is small for the full $56^{\circ}$ FOV. The deviation from true sun elevation angle (dashed red line) is probably due to a non-perfectly leveled tripod.

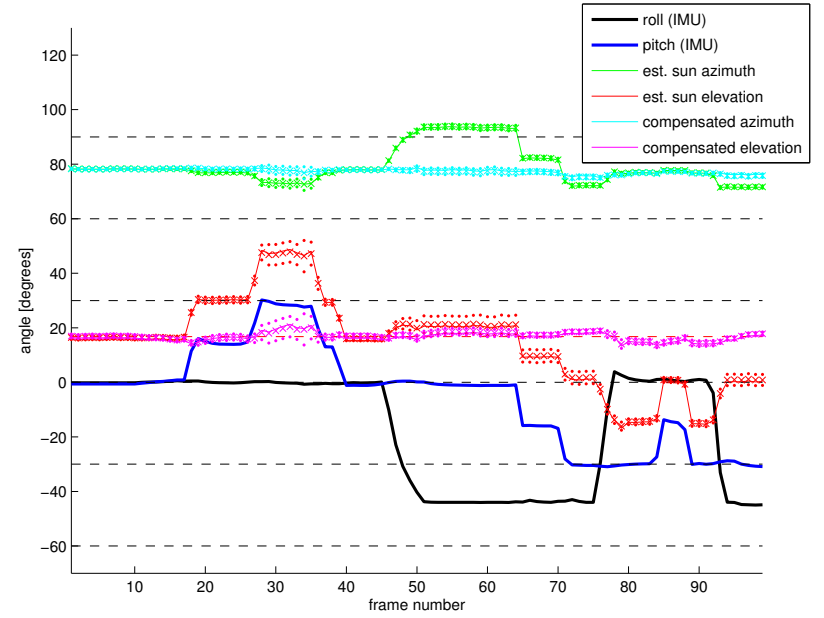

Figure 7. Estimation of sun azimuth and elevation angle and standard deviations for different tilt angles of the polarization sensor located under trees. Using the roll (black curve) and pitch (blue) angles measured by an IMU, the sun vector and its covariance matrix can be transformed into a leveled reference frame, Eqs. (45),(46), see curves and symbols in magenta for elevation and in cyan for azimuth angle. Note that possible errors due to IMU measurements are not considered.

Data was recorded under trees which, depending on the tilt angle, resulted in significant occlusion of the sky. The results of this experiment are shown in Fig. 7. Detailed examples corresponding to frame no. 1 (no tilt, large patches of clear sky) and frame no. 30 (tilt angle $30^{\circ}$, high level of occlusion) are illustrated in Figs. 8 and 9. As expected, the complex situation shown in Fig. 9 leads to uncertain estimates that are reflected in the larger size of the main axes of the error ellipse.

\section{Conclusion and future work}

The experiments have proved the polarization compass and the proposed direct method to be effective. It has also been shown that the system, if combined with an IMU, can provide accurate orientation information even when tilted. In future work, a tight integration of the polarization compass and an IMU in a filter-based framework will be realized, allowing to use the combined system on highly dynamic MAVs.

Acknowledgements. Special thanks to the mechanical workshop of the institute for producing the essential aluminium parts.

\section{References}

[1] H. G. Berry, G. Gabrielse, and A. E. Livingston. Measurement of the stokes parameters of light. Applied Optics, 16:3200-3205, 1977. 3

[2] J. Chahl and A. Mizutani. Biomimetic attitude and orientation sensors. IEEE Sensors Journal, 12:289-297, 2012. 1

[3] A. Cheung, S. Zhang, C. Stricker, and M. V. Srinivasan. Animal navigation: the difficulty of moving in a straight line. Biological Cybernetics, 97:47-61, 2007. 1 

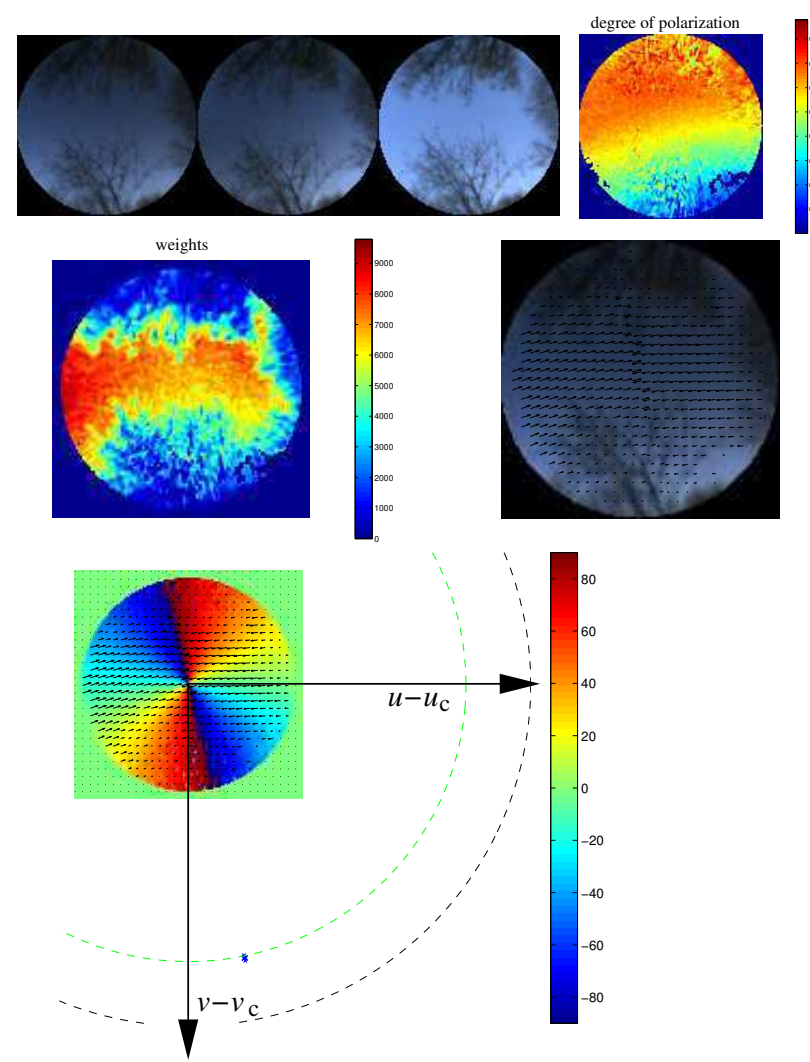

Figure 8. Sun vector estimation under trees with leveled camera (frame no. 1). Top row: Camera images (left); estimated degree of polarization (right). Second row: weight matrix (left); $x$ and $y$ components of estimated polarization vectors (shown for every 4 th pixel), their lengths scaled with local weights, mapped onto image of first lens (right). Bottom: sky polarization angles $\alpha_{i}$ with respect to great circles through the zenith, Eq. (13), in color code (blue $-90^{\circ}$, red $+90^{\circ}$ ), with polarization vectors overlayed; the blue cross in the lower right quadrant shows the estimated sun position and their covariance (principal axes scaled with square root of eigenvalues); the green circle marks the true elevation angle of the sun for the given location and date; the dashed black circle depicts the horizon $\left(0^{\circ}\right.$ elevation angle). The mapping onto the plane is defined by $u-u_{c}=\rho\left(\right.$ ele) $\cos ($ azi $), v-v_{C}=\rho($ ele $) \cos ($ azi $)$, where $\rho($ ele $)=\left(\frac{\pi}{2}-\right.$ ele $\left._{i}\right) / 0.5^{\circ}$, see Eq. (4).

[4] K. L. Coulson. Polarization and Intensity of Light in the Atmosphere. A. Deepak Publishing, 1988. 1

[5] D. D. Lambrinos, R. Möller, T. Labhart, R. Pfeifer, and R. Wehner. A mobile robot employing insect strategies for navigation. Robotics and Autonomous Systems, 30:39-64, 1999. 1

[6] M. Fleps, E. Mair, O. Ruepp, M. Suppa, and D. Burschka. Optimization based IMU camera calibration. In IROS, pages 3297-3304, 2011. 6

[7] P. T. Furgale, J. Rehder, and R. Siegwart. Unified temporal and spatial calibration for multi-sensor systems. In IROS, pages 1280-1286, 2013. 6

[8] R. Hegedüs, S. Åkesson, R. Wehner, and G. Horváth. Could
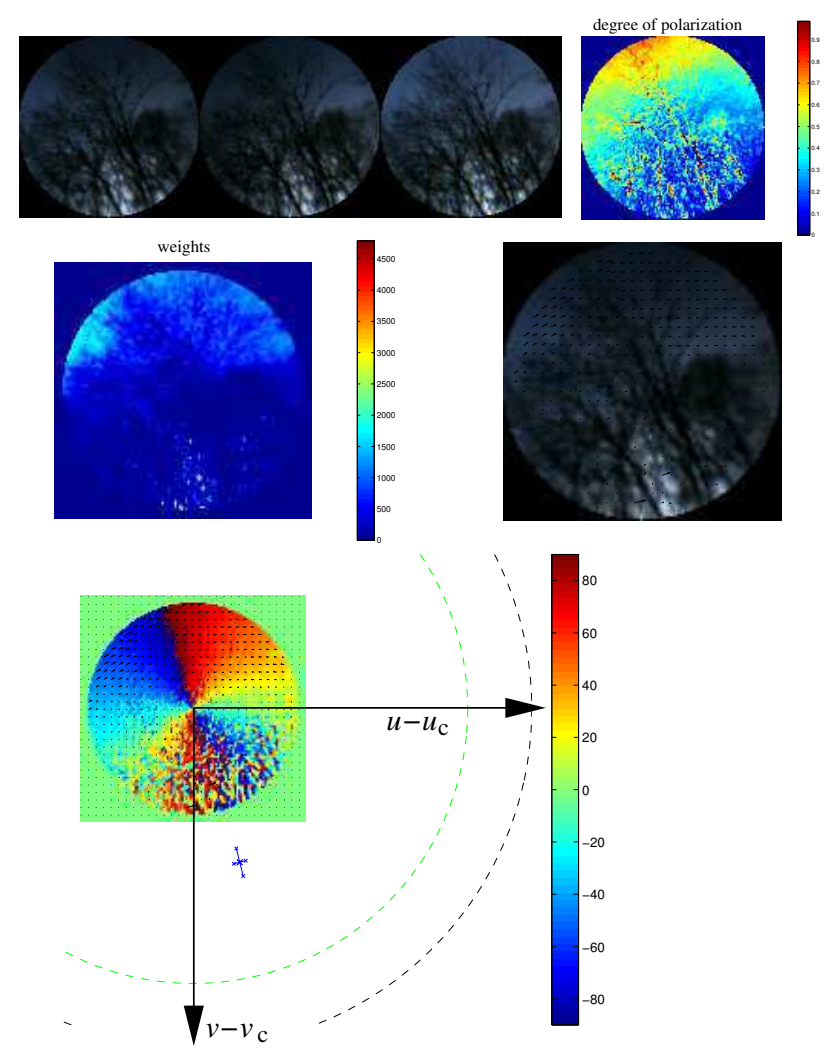

Figure 9. Sun vector estimation under trees with camera at about $30^{\circ}$ pitch angle (frame no. 30). Sub-figures as in Fig. 8. Note the smaller weights and the "noisy" pattern of the sky polarization angles. Also note that, compared to the situation shown in Fig. 8, the sun is now much closer to the center of the image due to the tilt of the camera, and that the estimated sun position error is much higher.

vikings have navigated under foggy and cloudy conditions by skylight polarization? On the atmospheric optical prerequisites of polarimetric viking navigation under foggy and cloudy skies. Proc Math Phys Eng Sci, 463:1081-1095, 2007. 1

[9] R. Horstmeyer, G. Euliss, R. Athale, and M. Levoy. Flexible multimodal camera using a light field architecture. In ICCP, pages $1-8,2009.1$

[10] G. Horváth, A. Barta, J. Gál, B. Suhai, and O. Haiman. Ground-based full-sky imaging polarimetry of rapidly changing skies and its use for polarimetric cloud detection. Applied Optics, pages 543-559, 2002. 1

[11] G. Horváth and D. Varjú. Light in Animal Vision: Polarization Patterns in Nature. Springer, 2004. 1

[12] S. B. Karman, S. Z. M. Diah, and I. C. Gebeshuber. Bioinspired polarized skylight-based navigation sensors: A review. Sensors, 12:14232-14261, 2012. 1

[13] R. L. Lee. Digital imaging of clear-sky polarization. Applied Optocs, 37:1465-1476, 1998. 1

[14] W. J. MacLean. Removal of translation bias when using subspace methods. In $I C C V$, pages 753-758, 1999. 4, 5 
[15] A. Manakov, J. F. Restrepo, O. Klehm, R. Hegedüs, E. Eisemann, H.-P. Seidel, and I. Ihrke. A reconfigurable camera add-on for high dynamic range, multi-spectral, polarization, and light-field imaging. In SIGGRAPH, 2013. 1

[16] F. Raudies and H. Neumann. A review and evaluation of methods estimating ego-motion. Comput. Vis. Image Underst., 116:606-633, 2012. 4

[17] M. Sarkar, D. S. S. Bello, C. V. Hoof, and A. J. P. Theuwissen. Integrated polarization-analyzing CMOS image sensor for detecting the incoming light ray direction. IEEE Trans. Instrum. Meas., 60:2759-2767, 2011. 1

[18] A. E. R. Shabayek. Combining omnidirectional vision with polarization vision for robot navigation. $\mathrm{PhD}$ thesis, Université de Bourgogne, 2012. 1

[19] K. H. Strobl, W. Sepp, S. Fuchs, C. Paredes, M. Smisek, and K. Arbter. DLR CalDe and CalLab, www.robotic.dlr.de/callab/. 2

[20] W. Stürzl and N. Carey. A fisheye camera system for polarisation detection on UAVs. In Computer Vision - ECCV. Workshops and Demonstrations, pages 431-440, 2012. 1, 4

[21] J. Weng, T. S. Huang, and N. Ahuja. Motion and structure from two perspective views: Algorithms, error analysis, and error estimation. IEEE Trans. Pattern Anal. Mach. Intell., 11:451-476, May 1989. 5

[22] S. Zhang, H. Liang, H. Zhu, D. Wang, and B. Yu. A camerabased real-time polarization sensor and its application to mobile robot navigation. In ROBIO, pages 271-276, 2014. 1

[23] W. Zhang, Y. Cao, X. Zhang, Y. Yang, and Y. Ning. Angle of sky light polarization derived from digital images of the sky under various conditions. Applied Optics, 56:587-595, 2017. 1 